\title{
AUTOMAÇÃO E DIGITALIZAÇÃO EM SUBESTAÇÕES DE ENERGIA ELÉTRICA: UM ESTUDO DE CASO
}

\section{AUTOMATION AND DIGITALIZATION IN ELECTRIC ENERGY SUBSTATIONS: A CASE OF STUDY}

\author{
Roberto Martins Pereira ${ }^{1}$; Ilda Maria de Paiva Almeida Spritzer ${ }^{2}$ \\ ${ }^{1}$ Federal Center of Technological Education - CEFET-RJ - Rio de Janeiro - Brasil rmp@ furnas.com.br \\ ${ }^{2}$ Federal Center of Technological Education - CEFET-RJ - Rio de Janeiro - Brasil spritzer@cefet-ri.br
}

\section{Resumo}

A automação de processos passou a fazer parte de nossas vidas. No cotidiano do cidadão comum, nos ambientes de chão de fábrica ou em grandes sistemas de transmissão de energia, o advento do microprocessador deu um impulso inimaginável às inovações tecnológicas em diversas áreas do conhecimento. O Setor Elétrico não é exceção. Este trabalho demonstra, através de um estudo de caso, como esses avanços se traduzem de maneira direta, ou seja, na utilização de Sistemas Integrados de Proteção e Controle pelas empresas de energia elétrica; e indireta, isto é, como essas empresas podem tirar proveito da tecnologia existente como ferramenta para aplicação dessas soluções. A revisão bibliográfica aborda os conceitos de automação e digitalização de sistemas de proteção e controle no âmbito das empresas de energia elétrica, bem como discorre sobre a evolução da microeletrônica nesses contextos. Considerações finais são tecidas a respeito das principais vantagens na utilização da tecnologia digital para implementação de funções de automatismo e telecomunicações.

Palavras-chave: Automação; Digitalização; Sistemas Integrados de Proteção e Controle.

\section{Introdução e Objetivos}

A solução de problemas de infraestrutura é condição necessária para que ocorra cidadania plena e para tanto, torna-se fundamental a garantia de acesso a serviços básicos, tais como: saneamento, transportes urbanos, comunicações e energia. Investimentos em ampliação e modernização da infraestrutura promovem a redução de custos, o aumento da produtividade, o aprimoramento da qualidade de bens e serviços da estrutura produtiva e a consolidação da integração regional, minimizando inclusive a possibilidade de interrupções em ciclos de crescimento do país. O Setor Elétrico representa um dos principais pilares para a garantia de um crescimento sustentável. Segundo Nivalde Castro (2006), coordenador do Grupo de Estudos do Setor Elétrico da UFRJ, o consumo de energia elétrica apresenta uma alta correlação com o desempenho da economia como um todo, pois praticamente todos os setores econômicos, que no 
somatório anual de sua produção definem a magnitude do PIB, têm um componente de consumo de energia elétrica.

O Setor Elétrico possui uma trajetória muito identificada com a busca constante por aperfeiçoamento tecnológico, alcançado em função de esforços continuados em Pesquisa, Desenvolvimento e Inovação. A crise de energia de 2001 demonstrou a vulnerabilidade do setor, e a necessidade de contínuo investimento na infraestrutura de geração e transmissão de energia elétrica existente, tendo como reflexo uma forte pressão sobre as empresas do setor, a fim de que garantam uma operação que otimize a segurança e a confiabilidade. Segundo Strachman (2005), a tecnologia tem sido crucial na reestruturação do Setor Elétrico, uma vez que as pressões de custos têm acrescido a relevância na melhora da performance, adicionalmente o controle dos fluxos de eletricidade tem motivado o interesse em sistemas de transmissão flexíveis. A utilização da tecnologia baseada em componentes eletrônicos de potência, proporciona maiores: velocidade, precisão e confiabilidade, do que as existentes nos componentes antigos.

Conforme citado por Paredes (2002), atualmente, os processos de manobra de sistemas de geração, transmissão e distribuição de energia elétrica utilizam-se da automação em suas atividades. Marquez (2003) destaca também uma crescente necessidade de Sistemas de Proteção tolerantes a falhas, com informações confiáveis para a garantia de uma operação segura.

A migração do paradigma analógico para o digital, nas áreas de proteção e controle das empresas de energia elétrica brasileiras, é apresentada neste trabalho por meio do estudo de caso específico de uma empresa do setor, no qual são apontadas etapas percorridas no processo de digitalização de suas subestações.

O trabalho visa ao aumento da compreensão sobre o papel das novas Tecnologias de Informação e Comunicação - TICs na implementação da automação em empresas do Setor Elétrico. A pesquisa sobre a implantação de Sistemas Integrados de Proteção e Controle de Subestações que compõem a rede de transmissão de $69 \mathrm{kV}$ e $138 \mathrm{kV}$ da empresa estudada permite vislumbrar algumas das principais vantagens da tecnologia digital na modernização dessas instalações.

\section{Automação e Digitalização}

Os avanços da microeletrônica na área industrial podem ser percebidos pelo desenvolvimento de sistemas de controle de máquinas cada vez mais sofisticados, combinando componentes mecânicos, pneumáticos, eletro-eletrônicos e ópticos. De acordo com Lastres e Ferraz (1997), o microprocessador é o fator-chave para o novo conjunto interligado de inovações em computação eletrônica, engenharia de software, sistemas de controle, circuitos integrados e telecomunicações. A aplicação da microeletrônica para automação de processos industriais adquiriu 
enorme importância, resultado do avanço dessas novas tecnologias. Em qualquer processo industrial, é vastíssima a utilização de esteiras e pórticos rolantes, motores, elevadores e demais equipamentos. A automação e o controle desses dispositivos, por meio das tecnologias atualmente disponíveis, proporcionam um significativo aumento da capacidade de produção. Em circuitos de comando de máquinas industriais mais complexas, observa-se a utilização em massa de Controladores Lógicos Programáveis (CLP's), Interfaces Homem-Máquina (IHM's), sensores magnéticos, sondas de temperatura, válvulas eletropneumáticas e demais. Toda a gama de novos equipamentos e sistemas de controle está marcada pela mudança do paradigma da utilização de componentes eletromecânicos para o novo paradigma dos equipamentos que utilizam eletrônica digital.

Para quem pudesse imaginar que essa revolução não se fizesse sentir nas empresas da geração e transmissão de energia elétrica, atualmente o Setor Elétrico experimenta semelhante migração dos sistemas de controle anteriormente baseados no paradigma analógico para o digital, o que, no âmbito dessas empresas, denomina-se genericamente "Digitalização de Subestações".

\section{Sistemas de Energia Elétrica}

O processo de geração, transmissão e distribuição de energia elétrica, pode ser analisado como um processo industrial. Inicialmente, ocorre a transformação dos insumos, sendo o principal a água, em um bem a ser fornecido: a energia elétrica. Na produção e no transporte de energia, não se utilizam esteiras ou elevadores, mas se faz uso de equipamentos tais como transformadores e disjuntores, que também precisam ser monitorados e controlados. Analogamente, a logística de distribuição e de entrega dessa mercadoria também não está baseada em caminhões, trens, aviões ou navios, estando sob a responsabilidade das empresas transmissoras e distribuidoras de energia, por meio de linhas de alta e extra-alta tensão, e de redes de distribuição que entregam o produto energia elétrica ao usuário final.

Segundo Caminha (1977), as empresas que se dedicam à tarefa de exploração de um sistema de energia elétrica, têm como meta:

- Garantir economicamente a qualidade do serviço;

- Assegurar uma vida razoável às instalações;

- Obter o melhor rendimento possível perante as perturbações e anomalias de funcionamento que afetam as redes elétricas e seus órgãos de controle.

Dessa forma, assumem uma importância crucial para essas empresas suas áreas responsáveis pela Operação (controle, supervisão e monitoramento) e pela Proteção do Sistema (contra curtocircuito e sobrecarga). 


\subsection{Proteção de Sistemas Elétricos}

Para o transporte de grandes blocos de energia elétrica, todo sistema de transmissão se baseia em redes de alta tensão, as quais requerem severidade máxima sobre a segurança de toda atividade inerente a sua exploração. No contexto das empresas de energia elétrica, o termo proteção pode assumir sentido de:

- Proteção das pessoas, tanto daquelas que trabalham como colaboradores dessas empresas nas atividades de operação e manutenção, bem como dos usuários da eletricidade em suas residências, ou ainda que morem ou trafeguem próximos de áreas energizadas (subestações, linhas de transmissão, postes e demais áreas);

- Proteções dos equipamentos constituintes de toda a cadeia de suprimento de energia elétrica, estejam eles nas usinas (ex: geradores), nas subestações (ex: transformadores) ou qualquer outro ponto do sistema. Os danos em equipamentos, principalmente dos que constituem o sistema de alta tensão, além de representarem enormes prejuízos financeiros para sua substituição ou reparo, podem ocasionar a interrupção do suprimento de energia em diversos níveis: ruas, bairros, cidades e até de um país inteiro, causando os chamados “apagões”, cujos prejuízos conseqüentes muitas vezes são de difícil mensuração;

- Uma terceira abordagem sobre este assunto, que é o foco do presente trabalho, diz respeito à proteção em um sentido mais amplo, de todo o sistema de geração-transmissãodistribuição (GTD), visando à preservação de sua capacidade de suprir energia ao consumidor final, com qualidade traduzida em reduzidos tempos de interrupção e freqüência de ocorrência dessas interrupções no serviço.

As duas principais características de um bom sistema de proteção são:

a) Segurança: o sistema deve ser capaz de operar nos casos em que seja estritamente necessário (ou seja, deve evitar a ocorrência de disparos intempestivos, que geram cortes desnecessários do fluxo de energia por determinado circuito);

b) Obediência: o sistema deve operar sempre que seja necessário (ou seja, não pode ser insensível à presença de fenômenos que possam ocasionar danos materiais ou risco à vida).

Os fenômenos que causam problemas na interrupção do suprimento de energia estão relacionados aos curtos-circuitos e às sobrecargas, que originam o que se denomina genericamente uma falta. No atual estado da arte, não existe equipamento capaz de evitar que ocorram faltas, mas sim de interromper, a altíssimas velocidades, os circuitos quando detectada a presença de uma falta. 
Os sistemas de proteção de sistemas de alta tensão têm como elemento-chave os dispositivos denominados relés de proteção. São esses os verdadeiros responsáveis pelos comandos de abertura dos disjuntores de uma subestação, e que permitem a extinção de uma falta, ou seja, do isolamento da falta, em velocidades que chegam a milésimos de segundo.

Os relés de proteção são equipamentos existentes há várias décadas, e construtivamente, sempre se basearam em dispositivos eletromecânicos e materiais magnéticos para integrar seus mecanismos de operação internos, usualmente comparados com mecanismos de relojoaria. Para a operação desejada dos relés de proteção em tempos tão rápidos, os avanços tecnológicos nesta área sempre estiveram relacionados com a pesquisa das propriedades magnéticas dos materiais.

\subsection{Sistemas de Controle - Evolução e Influências}

As subestações que constituem os sistemas de transmissão de energia elétrica são projetadas, sob o aspecto construtivo, com dois setores principais: pátio de manobras, onde se situam os equipamentos de alta tensão, e sala de controle, onde se localizam os Sistemas de Supervisão, Proteção e Controle da subestação. Desde a sala de controle se dá o monitoramento local dos estados das chaves e disjuntores, e de equipamentos importantes, tais como transformadores de potência e bancos de capacitores. Os quadros sinópticos convencionais já permitiam comandar remotamente, desde a sala de controle, por meio de chaves manuais, os equipamentos do pátio externo. Entretanto, a tecnologia até meados dos anos da década de 1970 baseava-se em dispositivos eletromecânicos, fazendo com que os quadros de comando ocupassem um considerável espaço físico da sala de controle da subestação.

Os avanços da aplicação da eletrônica analógica na área de automação e controle em sistemas produtivos industriais, no início dos anos 80, tornaram clara a possibilidade de seu uso ser estendido ao setor de energia elétrica. Pouco a pouco, as empresas deram seus primeiros passos e começaram a utilizar equipamentos eletrônicos nas salas de controle para aquisição de dados da subestação e envio remoto a Centros de Operação Regional (COR). Dessa forma, foi possível estabelecer o controle de todo um conjunto de subestações de uma região, permitindo a operação de unidades localizadas a grandes distâncias a partir de um mesmo computador central. Segundo Strachman (2005), a crescente importância dos fatores segurança e confiabilidade irão aumentar o mercado para sistemas de supervisão, controle e aquisição de dados baseados em computadores (SCADA, em inglês), que têm sido integrados a sistemas de controle e administração de sistemas de potência.

O dispositivo eletrônico formado pelo conjunto de unidades de aquisição, tratamento e envio de dados, localizado nas subestações denomina-se genericamente Unidade Terminal Remota - UTR, 
que representou num dado momento, uma grande revolução dentro de um setor reconhecidamente conservador na aplicação de novas tecnologias. Entretanto, a utilização daqueles dispositivos em larga escala ficou conhecida como "automação de subestações" equivocadamente; pois, em seu sentido estrito, esta denominação não é adequada, uma vez que a aplicação das UTR's permitiu tão somente o controle das subestações de forma remota por um sistema de nível central. Por outro lado, não deve ser desprezada sua importância, pois foi a partir da evolução da capacidade de transmissão de dados, através dos avanços nas telecomunicações, e da aplicação de computadores em escala ampliada, que surgiu o conceito de telecontrole de subestações. Possibilitando ampliar o potencial de utilização da tecnologia digital como uma realidade em ambientes de subestação.

Somente a partir dos anos 1990, a eletrônica, até então presente somente na operação dos sistemas elétricos, começou a penetrar o ambiente de proteção das empresas de energia elétrica, passando por dois momentos distintos e antagônicos:

- Primeira geração: relés eletrônicos baseados em tecnologia analógica - a experiência com esses equipamentos foi de certa forma, traumática para as empresas que se lançaram à frente na sua utilização, pois além de requererem ambientes com temperatura controlada, foram muitos os casos de "queima" de relés, e pior do que isso, de disparos indesejados desse tipo de proteção, reforçando a tese dos "conservadores";

- Segunda geração: relés baseados em tecnologia digital - pelo desenvolvimento da eletrônica digital e dos microprocessadores, essa geração foi muito bem sucedida, e representou uma mudança de paradigma na área, fazendo com que todas as empresas estejam realizando a migração de sua plataforma antiga formada por antigos relés eletromecânicos para a nova geração de relés digitais.

No final dos anos 1990, com a tecnologia eletrônica digital consolidada, surgiram as primeiras propostas de Sistemas Integrados de Proteção e Controle baseados nas seguintes condições:

- Predominância da eletrônica digital;

- Utilização de relés de proteção a base de microprocessadores e memórias;

- Utilização de terminais microprocessados integrando funções de controle e de proteção;

- "Inteligência local" em nível de equipamento para execução de lógicas e automatismos, tais como transferência de linha principal e transferência e esquemas de alívio e rejeição de carga, com o surgimento do conceito de IED - Dispositivo Eletrônico Inteligente. A Figura 1 representa um modelo esquemático com as principais funções desse tipo de dispositivo;

- Utilização de tecnologia de redes de computadores para comunicação por fibra óptica entre terminais e relés de uma mesma subestação; 
- Utilização de computadores pessoais para o nível de controle de subestação/sala de controle (nível 1-nível 2);

- Aquisição e envio de dados do computador central da subestação para o Centro de Operação (comunicação nível 2-nível 3).

Figura 1 - Dispositivos Eletrônicos Inteligentes - IED's

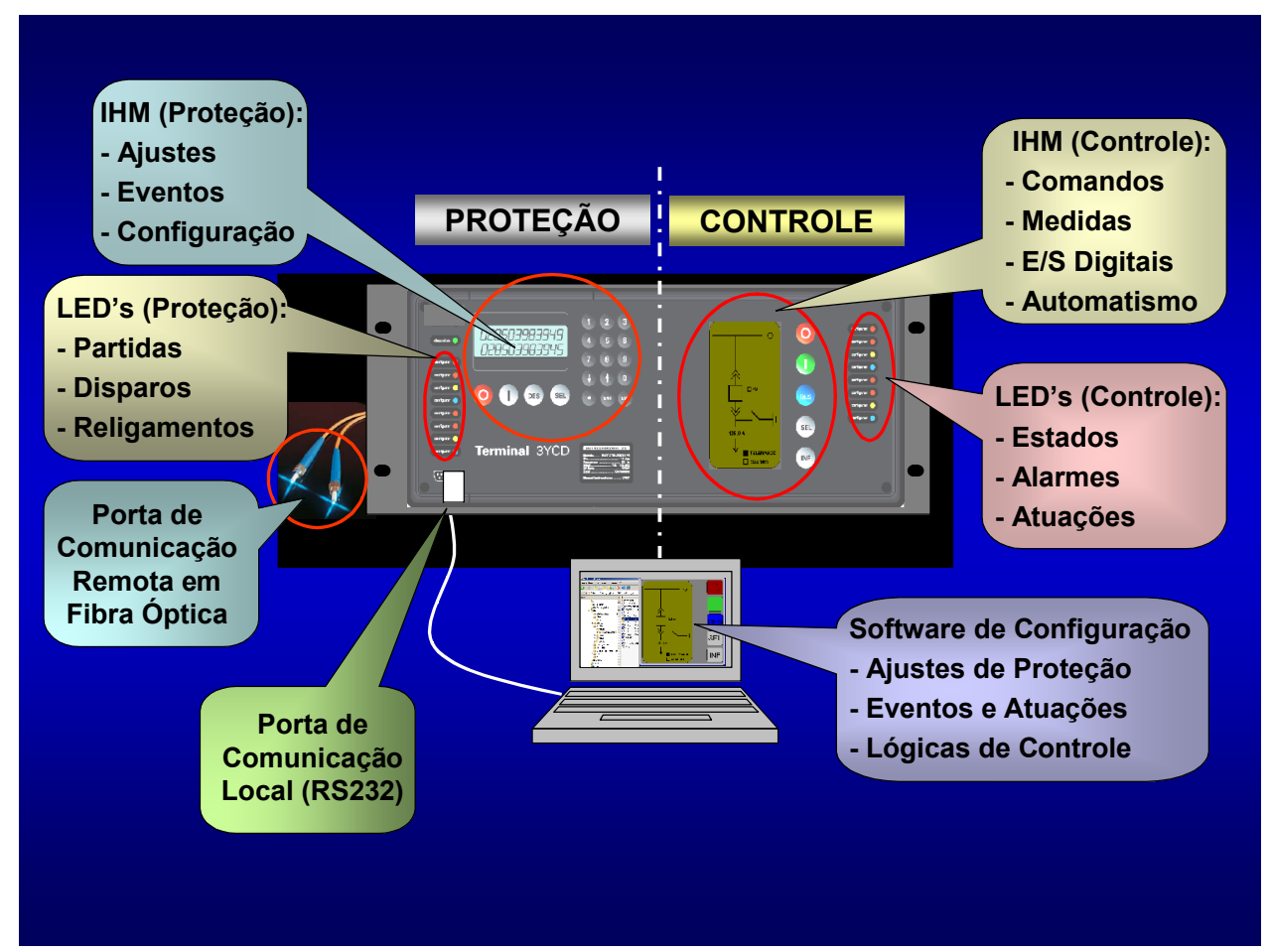

Fonte: Elaboração própria, 2006

Principais vantagens da nova geração:

- Maior precisão nas medidas;

- Maior rapidez de operação;

- Maior número de funções (de proteção) integradas;

- Maior flexibilidade de faixas de ajuste de proteção;

- Menor necessidade de manutenção (aumento do Tempo Médio Entre Falhas - MTBF);

- Maior confiabilidade (redução de partes internas móveis);

- Mais informação disponibilizada ao usuário;

- Maior capacidade de comunicação.

Além das características citadas, outras vantagens da digitalização de subestações, conforme citado por Pellini e Yamada (2001), e também observadas neste caso foram: topologia simples pela redução da quantidade de fíos e cabos na subestação para envio de dados, pelo uso de redes de fibra óptica; maior precisão na medida de grandezas analógicas; manutenção reduzida graças às rotinas de autodiagnóstico internas nos equipamentos; versatilidade nas funções, permitindo mudanças de 
faixas de ajuste de proteção, compensação de grupos de ligação de transformadores; e interoperabilidade, que é a capacidade de um dispositivo interagir com outros sistemas da subestação, composto por diferentes fabricantes.

Segundo Marquez (2005), após a mesma experiência de digitalização de subestações, sua empresa obteve redução de custos tanto do ponto de vista da instalação como de manutenção, devido entre outros fatores, à redução do número de relés, à simplificação do projeto o que se traduziu em um aumento da confiabilidade de seu sistema de transmissão de energia.

\section{Metodologia}

A apresentação de estudos de caso permite a verificação da aplicabilidade de teorias bem formuladas para o entendimento de diversas situações. Uma das vertentes que tem contribuído para a formulação de uma teoria para dar conta da evolução do paradigma tecno-econômico representado pela introdução da microeletrônica em diversas áreas da produção tem sido a corrente formada pelos Economistas do Aprendizado e da Inovação.

O presente estudo de caso individual realizado em uma empresa da área de distribuição de energia elétrica pretende contribuir para o aumento da compreensão das transformações que se têm observado no âmbito das empresas do setor elétrico.

Segundo Yin (2001, apud Rosal, 2004, p.36), este é o método "mais apropriado para estudos que procuram responder questões do tipo como e por que". Desta forma, partindo de uma empresa particular, procura-se evidenciar como a evolução tecnológica tem influenciado as empresas no processo de melhoria de seus desempenhos operacionais, ao mesmo tempo em que se torna mais visível o conjunto de fatores que justificam a adoção de tais medidas de caráter inovador.

\section{Estudo de Caso: Digitalização de Subestações de uma Empresa do Setor Elétrico Brasileiro}

Conforme citado anteriormente, as empresas de energia elétrica têm realizado investimentos no sentido de migrar a sua base instalada de equipamentos analógicos para a tecnologia digital. Neste trabalho, citamos um projeto já finalizado em uma concessionária de energia elétrica, a qual implantou um sistema de monitoramento e automação de sua rede de transmissão, digitalizando praticamente todas as suas subestações.

A empresa, tal como diversas outras do mesmo segmento de mercado, possuía um parque instalado de equipamentos já no final do período de sua vida útil, havendo a necessidade de modernização tanto sob o aspecto técnico como financeiro. A empresa não possuía experiências anteriores de utilização de dispositivos integrados de proteção e controle; por isso, antes de encomendar a substituição de todo o complexo composto por aproximadamente cem subestações, 
realizou uma experiência piloto em seis novas instalações. A partir do sucesso nesses empreendimentos, verificou-se a aplicabilidade do conceito para o restante do sistema e justificouse um investimento elevado para a modernização das subestações que já se encontravam em operação.

O empreendimento realizado pela empresa obteve êxito em diversos aspectos, dos quais destacamos como principais fatores de sucesso:

a) Âmbito do Fornecimento: em um primeiro momento, houve uma importante fase de definição da abrangência do escopo do fornecimento entre a contratante e seus fornecedores. Sob o ponto de vista dos fornecedores, essas reuniões favoreceram um melhor entendimento das necessidades e expectativas de seu cliente e uma definição de padrões de projeto para todas as instalações da concessionária. Por outro lado, ao longo dessas reuniões, a contratante foi percebendo mais detalhes sobre as possibilidades, que os novos equipamentos digitais permitiam que ao final dessa etapa, já estivesse definida uma base de dados, projetada sob equipamentos com lógica personalizada para as especificações técnicas requeridas;

b) Projeto: pelo uso de equipamentos (relés de proteção e terminais integrados tipo IED) e projeto padronizados, houve enorme economia de tempo tanto para sua fabricação e montagem pelos fornecedores, como para a sua efetiva implantação em campo: a padronização de equipamentos (terminais de proteção e controle) foi possível pela definição dos modelos a serem utilizados para cada trecho (vão) da subestação, ou seja, para os vãos de linhas de transmissão, onde se utilizavam as funções de proteção de distância e direcional; para os vãos de transformador, onde se aplicaram relés com funções de proteção diferencial, de sobrecorrente e de proteção intrínseca; e para os vãos de alimentadores de média tensão e de bancos de capacitores, para os quais se definiu a proteção de sobrecorrente. Com relação à padronização de projeto, foram definidas lógicas de controle nos terminais, tais como esquemas de falha de disjuntor, regulação de tensão e acionamento de grupos de ventilação nos transformadores, de tal forma que o hardware disponibilizasse entradas e saídas digitais com a mesma codificação para subestações diferentes;

c) Rapidez na Implantação: na maior parte do tempo, os trabalhos foram realizados com o sistema de transmissão em uso (subestação energizada), o que não representou diminuição em seus índices de qualidade de fornecimento de energia elétrica: toda vez que se interrompe o suprimento de energia, os indicadores de qualidade de uma empresa de energia elétrica caem. Para este tipo de empreendimento, tradicionalmente, são previstas muitas interrupções no sistema, para que as equipes de campo trabalhem com 
equipamentos desenergizados. Entretanto, o tempo para os testes de comissionamento utilizando este novo tipo de sistema integrado foi reduzido drasticamente, o que fez com que a equipe responsável pelo projeto desenvolvesse um sistema de trabalho conjunto no quais os desligamentos foram minimizados e programados para horários de menor impacto, tais como feriados e finais de semana. A Figura 2 representa um exemplo da funcionalidade obtida pela implantação de ajustes em equipamentos situados no pátio das instalações por meio de um computador portátil do tipo notebook;

d) Independência entre níveis: a substituição dos sistemas anteriores proporcionou à concessionária de distribuição acesso em tempo real às informações acerca de todas as suas subestações; com a possibilidade de exercer o comando remotamente, a partir do Centro de Operação do Sistema. Por outro lado, ao utilizar o conceito de "inteligência local", o controle da subestação, por meio das lógicas instaladas em seus terminais de controle e proteção locais não dará lugar à ocorrência de qualquer desligamento inoportuno ou não-atuação, mesmo com uma possível perda de comunicação entre esses dois níveis.

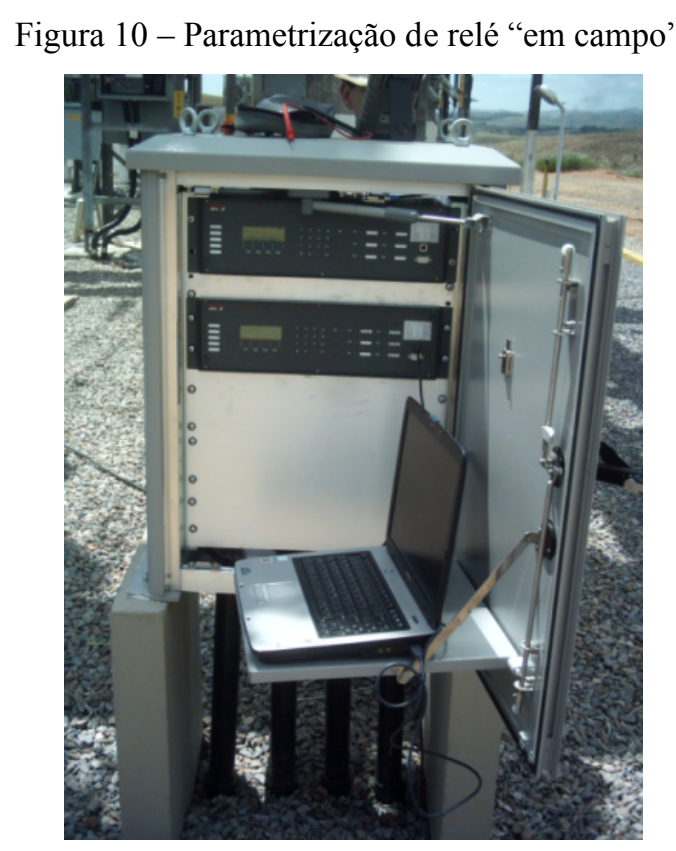

\subsection{O Papel das TIC's na Implementação dos Sistemas Integrados}

Além de toda a riqueza de dados e informações que os novos Sistemas de Controle e Proteção colocam à disposição do usuário, por meio dos, é particularmente importante a forma como a tecnologia dos equipamentos microprocessados, serviu para se atingir o êxito deste projeto, reduzindo em grande parte os tempos normalmente necessários para a implantação de 
empreendimentos similares. As principais atividades relacionadas com a implementação da modernização das subestações foram:

a) Disponibilização dos diagramas unifilares das subestações pela concessionária;

b) Verificação, por equipes de campo, das atualizações (“como construído") para a correção dos diagramas disponibilizados;

c) Definição das novas lógicas de controle e das funções de proteção a serem previstas para cada tipo de terminal IED;

d) Envio das lógicas definidas na etapa anterior para a empresa contratada para configuração e validação em laboratório, usando correio eletrônico;

e) Utilização de aplicativo (software) para criação das lógicas de controle;

f) Utilização de vídeo e audio-conferências para discussão de eventuais dúvidas ou discordâncias entre a concessionária e as empresas contratadas;

g) Utilização da internet para acesso aos arquivos a serem inseridos nos terminais IED por meio de notebooks. Em alguns casos, este acesso pôde ser feito inclusive, desde a própria subestação onde o especialista estava implementando as configurações dos terminais;

h) Utilização de portas de comunicação existentes nos novos relés e terminais de proteção e controle para inserir configurações de lógicas e ajustes de proteção por meio de protocolos de comunicação específicos;

i) Testes operacionais em campo das configurações de controle e ajustes de proteção com o auxílio de equipamentos de testes, tais como fontes de grandezas analógicas e atuação sobre os equipamentos de campo para verificação das funções de controle e monitoramento.

\subsection{Vantagens Obtidas com a Digitalização}

Destacam-se abaixo alguns dos principais êxitos alcançados pela empresa de distribuição de energia quando da implantação dos Sistemas Integrados de Proteção e Controle em suas subestações.

- Redução do espaço físico requerido nas salas de controle para alocar os sistemas de controle e de proteção, confirmando estudos semelhantes, como os de Pellini e Yamada (2001);

- Manutenção da continuidade do serviço durante todo o período de transição para o sistema novo;

- Tempo para migração para o novo sistema drasticamente reduzido, pela utilização de tecnologias de informação e comunicação (TIC's), tais como compartilhamento de desenhos, softwares e lógicas de controle; 
- Acesso à informação sobre o sistema de transmissão da empresa em tempo real, permitindo uma melhoria substancial na qualidade da tomada de decisão no caso de necessidade de manobras e de análise de atuação do sistema de proteção (melhoria na confiabilidade dos dados);

- Redução de gastos com operadores de subestação, pela concepção de subestação desassistida (melhoria da eficiência da empresa);

- Modernização do parque instalado, elevando o valor dos ativos da empresa;

- Padronização dos equipamentos utilizados, facilitando a gestão de estoque e de compras futuras.

\section{Considerações Finais}

Foi apresentado o conceito de automação e a forma como a evolução de sua aplicação no âmbito industrial influenciou as empresas de energia elétrica, tradicionalmente mais conservadoras na utilização de equipamentos de tecnologia microeletrônica em suas instalações.

O caso apresentado de aplicação dos sistemas integrados demonstra que os novos equipamentos baseados em eletrônica digital e tecnologia de informação, além de agregarem um número amplamente maior de funções, permitem a realização de lógicas locais (automatismos) e envio/acesso de dados remotamente, ao Centro de Operação de Sistema (COS).

A implantação dos novos Sistemas Integrados de Proteção e Controle pode ser considerada plenamente satisfatória na empresa apresentada. Para as empresas que estão começando o processo de digitalização de suas subestações, podem ser destacados alguns aspectos relevantes na implantação de projetos deste porte, tais como o investimento no quadro de colaboradores para a discussão prévia dos requisitos técnicos do projeto desde a fase inicial; o desenvolvimento de uma política de recursos humanos que potencialize novas atribuições para o pessoal da operação; o contato com empresas que tenham adquirido experiência em projetos similares; o desenvolvimento da capacitação crítica dos gestores que optem por este tipo de solução para que seja capaz de avaliar, durante todas as etapas do projeto, a maneira mais adequada para realizar esta migração, pelas características próprias de suas empresas, tendo sempre a consciência de que qualquer processo de mudança de paradigma envolve grandes esforços organizacionais.

\footnotetext{
Abstract

The automation of processes has become an usual issue of our lives. In common activities of the ordinary citizen, in plants sites environments or in the great energy transmission systems, the incoming of the microprocessor has given unimaginable impulse to the technological innovations in several areas of the knowledge. The Electric Industry is not an exception. This paper demonstrates, by means of an study of case, how those advances are translated either directly, when used in the
} 
Protection and Control Integrated Systems by the electrical firms, or indirectly, it is, how these firms can take advantage from the existent technology as a tool for the application of those solutions. The bibliography approaches some concepts such as automation and digitalization of protection and control systems in the scope of electric energy companies and also brings information about the evolution in microelectronics on those contexts. Final considerations are woven regarding the main advantages obtained in the use of digital technology for the implementation of automation and telecommunication functions.

Key-words: automation, digitalization, protection and control integrated systems.

\section{Referências}

CAMINHA, A. C.; Introdução à Proteção de Sistemas Elétricos. Editora Edgard Blucher Ltda. $7^{\text {a }}$ Edição. São Paulo, 1977.

CASTRO, N. J.; BRUNI, P .P. B.; A Dinâmica da Oferta e Demanda de Energia Elétrica no Brasil em 2005-2006. ELETROBRAS, 2006

LASTRES, H. M. M.; ALBAGLI, S.; Informação e Globalização na Era do Conhecimento. Editora Campus, 1999.

MARQUEZ, D. C.; KRAUSS, C. C.; FRISCH, A. C.; PAROLIN, E. L.; A Experiência da Copel na Integração de Proteções Digitais ao Sistema de Automação de Subestações. Seminário Nacional de Produção e Transmissão de Energia Elétrica - XVII SNPTEE. Uberlândia, 2003.

PAREDES, A. E. R. O.; Integração de Sistemas de Supervisão, Proteção e Automação de Subestações de Energia Elétrica. Dissertação de Mestrado. Universidade Federal de Itajubá, 2002.

PELLINI, E. P.; YAMADA, P.; Digitalização de Subestações: conceitos, vantagens e aplicações. Revista Controle \& Instrumentação. Edição 58. São Paulo, 2001.

ROSAL, A. C. L.; Trajetória de Desenvolvimento Tecnológico na Indústria de Transmissão de Energia Elétrica: a experiência das Centrais Elétricas do Norte do Brasil S.A. - ELETRONORTE. Dissertação de Mestrado, FGVEBAPE, Rio de Janeiro, RJ, Brasil, 2004.

STRACHMAN, E.; Relatório Setorial: Equipamentos Elétricos sob Encomenda. Diretório da Pesquisa Privada. Arquivo capturado em 26/05/2006 em <http://www.finep.gov.br>. FINEP, 2005.

\section{Dados dos autores:}

Nome completo: Roberto Martins Pereira

Filiação institucional: FURNAS CENTRAIS ELÉTRICAS S.A

Departamento de Engenharia Elétrica

Cargo ocupado: Engenheiro de Proteção, Controle e Supervisão

Endereço completo: Rua Real Grandeza, 219 - Bloco C / Sala 301 - Botafogo-RJ

Telefones: (21) 2528-2516

E-mail: rmp@furnas.com.br

Nome completo: Ilda Maria de Paiva Almeida Spritzer

Filiação institucional: CEFET-RJ

Departamento: Diretoria de Pesquisa e Pós-Graduação

Função ocupado: Professor Adjunto 
Endereço completo: Av. Maracanã, 229-bloco E - $5^{\circ}$ andar

Rio de Janeiro - RJ, CEP: 20271-110

Telefones: 21-2569-4495

E-mail: spritzer@cefet-rj.br

Recebido para publicação em: 10/11/2007

Aceito para publicação em: 06/12/2007 